\title{
Ethical Challenges of International Outbreaks-A Case Study of Ebola Virus Disease
}

\author{
Article by, Okafor $\mathrm{CN}^{1}$, Nwobi $\mathrm{AE}^{2}$, Anyaehie $\mathrm{BU}^{3}$, Young $\mathrm{EE}^{4}$ \\ ${ }^{1,3}$ Department of Physiology, University of Nigeria, Enugu Campus \\ ${ }^{2}$ Department of Community Medicine, University of Nigeria, Enugu Campus \\ ${ }_{4}$ Department of Medicine, University of Nigeria, Enugu Campus \\ E-mail: ${ }^{1}$ chinyere.okafor@unn.edu.ng, ${ }^{2}$ amaebie@yahoo.com, \\ 3bond.anyaehie@unn.edu.ng, ${ }^{3}$ ekenechukwu.young@unn.edu.ng
}

\begin{abstract}
Public health measures are strictly required to prevent increase in the spread of Ebola virus disease and that includes identifying cases and quarantining persons who are suspected to have come in contact with the disease or its agent. Though quarantine restricts freedom of movement of those affected, the reasons may be justified based on the fact that health of the public needs to be protected. But though the personal rights and liberties of individuals may be expected to be lost in international outbreaks, it should not be totally taken away. There ought to be a balance between preserving individual rights and maintaining public health. Thus a legal and political framework is needed while research for therapy and vaccine are encouraged.
\end{abstract}

Keywords: Ebola virus disease, ethical challenges, international outbreaks.

\section{Introduction}

An infectious disease such as Ebola virus disease can threaten the stability of a country's or region's government, economy, and social fabric. ${ }^{1}$ Ethics is concerned with distinguishing between good and evil in the world, between right and wrong human actions, and between virtuous and non-virtuous characteristics of people. ${ }^{2}$ It can be further defined as the rules of conduct recognized in respect to particular class of human actions or a particular group, culture, etc. ${ }^{3}$ Despite the existence of ethics guidance in relation to specific diseases (e.g. pandemic flu, TB and HIV) comprehensive international/global public health ethics guidance related to epidemic outbreaks is lacking ${ }^{4}$. Ethics does not provide a prescribed set of policies; rather, ethical considerations will be shaped by the local context and cultural values. The response to any outbreak entails being prepared to limit individual rights and civil liberties in the interest of public health. International outbreaks have increased the social and economic inequalities within and between countries with its economic consequences. The recent Ebola outbreak has presented a number of ethical issues some of which are new especially due to the cultural and political atmosphere in affected countries leading to significant challenges for health care workers and public health officials trying to contain the epidemic. Some deny that Ebola is real, even going so far as to claim that there is no epidemic, with claims that the government health workers are killing patients to simulate an epidemic and receive Western funding ${ }^{5}$. Those who are ill stay away from the hospitals for fear of becoming infected with Ebola; those who have Ebola hide to avoid stigmatization ${ }^{5}$.

The last epidemic of Ebola virus disease is one of two dozen outbreaks since discovery of the virus in $1976^{5}$. It was identified along the border of Guinea and Sierra Leone March, 2014 subsequently spreading to Liberia, with a handful of cases in Nigeria associated with the arrival of an infected airline passenger ${ }^{6}$. As of October 14, 2014, a total of 8,914 probable, confirmed, and suspected cases of EVD and 4,447 deaths due to the disease, have been reported to the World Health Organization (WHO). The WHO estimated then that by December 2014, the number of new EVD cases could rise ten-fold, to 10,000 per week, and the survival rate in these West African countries was "30 percent at most"?. 
The virus is transmitted in body fluids, like blood, saliva, vomit or stool but not through the air. Caregivers of infected patients, and those who prepare them for burial, are at particular risk $^{5}$. Recent reports which states that the virus appears to be showing genetic mutations, even during the current epidemic is a source of worry. The African derived Ebola virus infections, there is an incubation period that can last from three days to three weeks ${ }^{5}$. The virus is not spread until the patient becomes symptomatic with fever, chills, headache, muscle aches, vomiting and diarrhea ${ }^{5}$. Later they develop a hemorrhagic rash, and bleeding from any mucous membranes.

Approved specific therapy for treatment is not available yet as well as vaccine for prevention. Thus intensive general medical support is very crucial for survival, with Intra Venous fluid therapy and measures for controlling bleeding. This care must be given with strict attention to barrier isolation from the contaminated fluids from the patient as well as to provide the best likelihood of survival for the infected patient ${ }^{5}$. Protective barriers for healthcare workers (gloves, gowns, masks and goggles) should be worn for identified Ebola cases $^{5}$. However, identification may be delayed because the early symptoms can resemble malaria, typhoid fever, cholera, and other illnesses which present much more commonly in the African geographic region ${ }^{5}$.

Government outbreak response activities to control the spread of Ebola has involved isolation, restriction of free movement and liberty. It is against this background that the public have raised ethical concerns and argued that outbreak response activities conflict with the constitutionally protected rights of individuals with suspected and probable Ebola cases ${ }^{8}$.

In this study, we wish to identify the ethical challenges involved in international outbreaks using Ebola virus disease as case study and proffer solutions to these ethical challenges experienced during international outbreaks.

\section{Methodology}

Information was obtained by a desk review of publications and reports on the recent Ebola virus disease outbreak and ethical challenges involved, by Center for Disease Control, World Health Organization etc. A detailed literature search was done to identify articles on the management of the recent Ebola virus disease outbreak, and to identify ethical issues experienced by health care workers and public health officials.

\section{Discussion}

The recent EVD outbreak was an international public health crisis ${ }^{9}$. The number of legal and ethical questions arising out of prevention, treatment, and public health response to EVD can be divided into social, medical, economic and psychological aspects and all are interwoven.

\section{Medical issues}

\section{The use of experimental drugs in international outbreaks without consent}

In this issue, the benefits and dangers involved need to be considered. As in the case of EVD, the drugs that had never been tested in human subjects were given to sick patients as there was no time to do the necessary clinical trials. So far they have only been tested in a handful of monkeys and first phase not yet completed in human beings. Thus the adverse effects were not known and the drugs could not be said to be safe or effective. For example, of the 6 people who received an experimental therapy of Z Mapp, 2 have died. This doesn't prove its efficacy or safety as long term effects cannot be assessed. On August 11, 2014, the World Health Organization convened a panel to discuss these ethical issues. Their response was widely reported: "It is ethical to offer unproven interventions with as yet unknown efficacy and adverse effects, as potential treatment or prevention"7. 


\section{Who should be treated as there are always scarce resources in international outbreaks?}

The code of ethics for medical practitioners in Nigeria and other parts of the world calls on medical practitioners to first consider the well-being of their patients. But this ethical duty must be balanced with a duty not to become a source of harm to other members of the public which would likely be the case where the medical practitioner himself gets infected. Thus healthcare workers should be treated first in outbreaks so that they will not be a source of transmission of infection to the public and also be healthy enough to combat the number in the outbreaks. Also healthcare workers are most likely to enroll in trials of experimental therapies and understand the requirements of informed consent in the treatment especially in African setting with various cultural misinformation.

A lack of resources for the diagnostic and supportive services required to manage EVD is another key issue ${ }^{10}$. The absence of diagnostic kits means that the quarantine period is lengthened, as a result of which people's rights of movement are curtailed and their ability to earn is limited for periods much longer than if they had been tested during contact tracing and declared Ebola-free ${ }^{10}$. The lack of adequate protective gear as well as other healthcare items, such as gloves and single-use syringes, is also a matter of ethical concern ${ }^{10}$. The risk to which the healthcare and ancillary workers took which as well as took some lives of doctors in Nigeria who later contracted EVD while saving lives with the few protective items available are also ethical challenges that could have been avoided. Lives of health workers could have also been saved if vaccines and therapeutics to counter EVD had been available.

\section{Social and Economic aspect}

During international outbreaks as in EVD, quarantine and isolation, are often employed and this are very restrictive of personal rights especially right to liberty and freedom of movement. For example in Nigeria, many of the subjects who came in contact with an infected patient were quarantined for a very long time though they were later found to be Ebola-free and this lead to increased stigmatization from their relatives and the public. This also resulted in other contact subjects not reporting to the hospital and also not disclosing their infected relatives.

They also impact on the income earning ability of the persons under isolation e.g. during Ebola outbreak in countries like Liberia and Sierra Leone, government placed community quarantine and a three day nationwide curfew respectively and this led to limitation of movement leading to food shortages, poor access to essential services including health, social limitation and economic enhancement. The absence of a framework which ensures that the persons under quarantine do not lose any economic benefits that they may be entitled to might deter persons who suspect themselves to have been exposed to the disease agent from disclosing it.

\section{Psychological and emotional aspect}

In international outbreaks, response activities like isolation, restriction of movement affect the individuals psychologically. Using Nigeria EVD patients as example, the individuals felt neglected, isolated and abandoned with no personal contact, family or friends amidst the suffering. They became anxious, felt hopeless and helpless and with depression developing among confirmed, suspected and likely cases. During these outbreaks, medical practitioners are put under an obligation to report all cases of persons who harbor any illness or health condition that may be similar to these epidemics for isolation from the rest of the public. As a result of no emotional support individuals develop stress related disorders like ulcers, schizophrenia and posttraumatic stress disorder among the isolated cases.

The family contacts of EVD victims go through the dilemma of whether to abandon or continue to care for the victim. They live in great fear and psychological torture during the quarantine period. Relatives like spouses of EVD survivors also live in fear of possible 
Texila International Journal of Public Health

Volume 4, Issue 4, Dec 2016

continued sexual transmission of the virus after recovery and future generations yet unborn being affected.

\section{What are possible ways of combating these challenges?}

The recent control Ebola Virus Disease (EVD) in Nigeria was globally regarded as effective $^{11}$. Although there might be restrictions of individual rights, a procedural framework would help provide for any such restrictions to be minimal. This envisages that measures taken for prevention of spread of virus must also help reduce some of these ethical issues. The measures used included the following;

1. The individual must have actually been exposed to the infectious agent and thus pose an actual threat to the public as contemplated by the Nigerian Constitution.

2. The measures taken for restriction must be reasonable and effective and no discrimination must be employed.

3. The government should help provide diagnostic and supportive services and also protective wears to help the healthcare workers in management like in Nigeria were funds were generously allocated and isolation facilities built. Also infrastructures and cutting-edge technologies used for our global outbreaks were repurposed to support Ebola response $^{11}$.

4. Individuals involved should be provided with a choice, where practicable, of whether to be quarantined in a government run facility or in their own homes with rules to ensure compliance.

5. The measures taken must ensure reasonable compensation for loss of income due to the quarantined individual's inability to go to work so as not to infect others.

6. There must be adequate measures for integrating the persons who have been placed on quarantine or isolation back into the society and manage any stigmatization that may occur.

7. There is a critical need for capacity building in the ethical review of public health research and practice. Researchers, public health agencies and other stakeholders should work together to develop short courses, degree programs and other training modalities. Funding agencies should direct appropriate support to these efforts ${ }^{7}$.

8. Actions towards the invention of vaccine and drugs for treatment should be taken more seriously.

Transparency in handling public engagement with the involvement of relevant stakeholders was a cardinal pathway in the Nigerian experience. Increased public awareness about the disease and its related risks enabled people to take steps at individual, family, workplace and community level to prepare for and respond to an epidemic. Nigeria deployed all social media platforms in the fight. ${ }^{12}$. GPS systems were put to work for real-time contact tracing and daily mapping of transmission chains and contact tracing reached 100\% in Lagos and $99.8 \%$ in Port Harcourt $^{11}$. However, the use of multiple platforms by multiple groups in an uncoordinated way provided gaps in ethics.

\section{Limitations to some of these measures}

In some of the social media platforms like WhatsApp there was no censorship of what was posted and there was no punishment for wrong information by persons. This lead to lack of transparency as multiple news were being given. There is always a need to promote public compliance and mitigate fears. This led to people ingesting all kinds of alternative medications like salt water, herbs, etc.

Confidentiality which is the obligation to keep information secret unless its disclosure has been appropriately authorized by the person concerned or, in extraordinary circumstances, by the appropriate authorities was missing. 


\section{Conclusion}

This outbreak has demonstrated, like nothing before, that in today's world ${ }^{10}$ - which is characterized by interconnectedness, increasing globalization and easier means of transportation, and in which pathogens no longer respect the borders of countries - no country can remain immune to the risk of pathogens, whatever their origin, whether it is the jungles of Africa or a metropolitan city of India. Countries should, therefore, make it a priority to strengthen their health systems to manage health and disease in normal times as well as in times of emergency ${ }^{10}$. Finally, national preparedness plans for the control of epidemics are needed.

\section{Figures and tables}

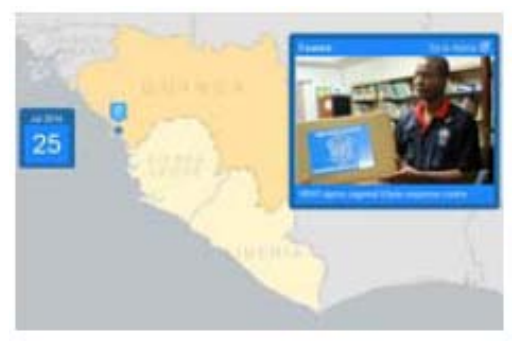

Fig 1. 2014 West Africa Ebola outbreak

Source WHO 2014

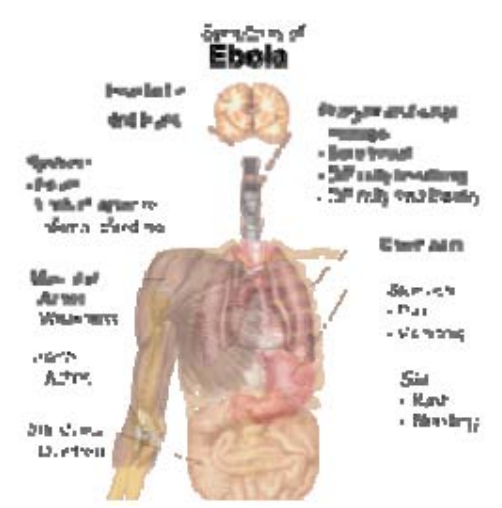

Fig 2. Signs and Symptoms of Ebola Virus Disease

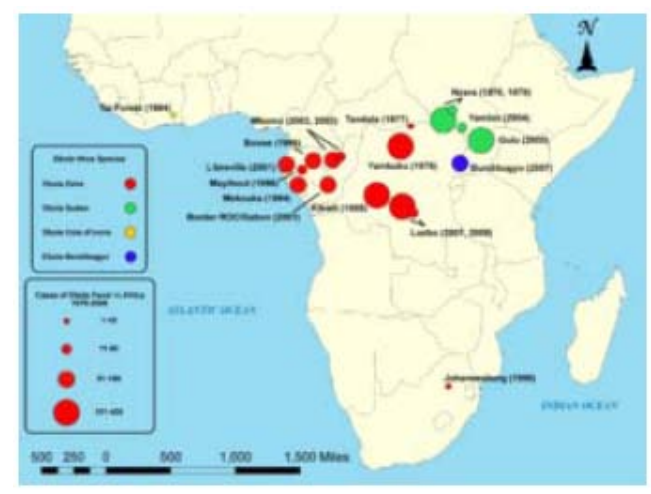

Fig 3. Cases of Ebola fever in Africa from 1979 to 2008 
Texila International Journal of Public Health

Volume 4, Issue 4, Dec 2016

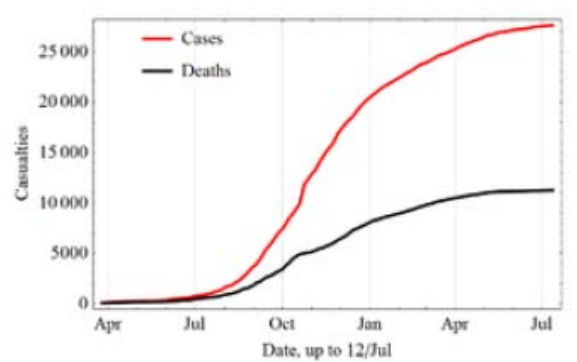

Fig 4. Increase over time in the cases and deaths during the 2013-2015 outbreak

Source Ebola Virus epidemic in West Africa

\section{References}

[1] Abha S. Ebola Virus Disease Outbreak- Incorporating Ethical Analysis into the Health System Response. Indian Journal of Medical Ethics 2014, Vol 11; 4. http://www.who.int/csr/disease/ebola/one-year-report/nigeria/en/

[2] Center for Disease Control and Prevention: Ebola (Ebola Virus Disease): 2014 Ebola Outbreak in West Africa. Atlanta, Georgia; 2014 [http://www.cdc.gov/vhf/ebola/outbreaks/2014-westafrica/index.html].

[3] Ebola Virus Disease- Legal and Ethical considerations for Indiana. Improving Community Health through Policy Research, Center for Health Policy 2014.

[4] Global Forum on Bioethics in Research (GFBR). Meeting report: Emerging epidemic infections and experimental medical treatments. Annecy, France. 3-4 November 2015. Available from: http:/www.gfbr.global/wp-content/uploads/2016/03/GFBR-2015-meeting-report-emergingepidemicinfections-and-experimental-medical-treatments.pdf (Accessed October 22 2016).

http://www.dictionary.com/browse/ethics

[5] Ibrahim W. Ethical concerns regarding Ebola control measures in Uganda, Makerere University School of Public Health 2013.

[6] Kevin G. Ebola, Epidemics and Ethics- what we have learned. Donovan philosophy, Ethics and Humanities in Medicine 2014, 9:15.

[7] Nduka UC, Igwe-Omoke A, Ogugua C. The use of social media in combating the Ebola virus in Nigeria- a review. International Journal of Medicine and Health Development.2014 19 (1/2): 97-108

[8] Osterholm MT, Moore KA, Gostin LO. Public Health in the Age of Ebola in West Africa. JAMA Intern Med. 2015; 175(1):7-8.

[9] Pan American Health Organization. Zika Ethics Consultation: Ethics Guidance on Key Issues Raised by the Outbreak. Washington, DC: PAHO, 2016. Document Number: PAHO/KBR/16-002. Available

at http://iris.paho.org/xmlui/bitstream/handle/123456789/28425/PAHOKBR16002_eng.pdf (Accessed October 22 2016).

[10] WHO (2010). Research Ethics in International Epidemic Response, Geneva 2009. 